\title{
Incidencia creciente en el carcinoma de células renales
}

\author{
J.M. Giménez Bachs, MẫJ. Donate Moreno, A.S. Salinas Sánchez, J.G. Lorenzo Romero, \\ M. Segura Martín, I.R. Hernández Millán, H. Pastor Navarro, B. Martínez Córcoles, \\ L. Cañamares Pabolaza, J.A. Virseda Rodríguez
} Servicio de Urología. Complejo Hospitalario Universitario de Albacete. Albacete.

\section{RESUMEN}

INCIDENCIA CRECIENTE EN EL CARCINOMA DE CÉLULAS RENALES

Objetivos: Analizar el cambio en el comportamiento del carcinoma de células renales en cuanto a su modo de presentación, tratamiento, anatomía patológica y mortalidad durante un periodo de 17 años.

Material y método: Estudio retrospectivo sobre 212 pacientes intervenidos en nuestro Servicio por carcinoma de células renales desde el año 1988 hasta 2004, analizando los datos clínico-demográficos y comparándolos entre sí según dos periodos: 1988-1996 y 1997-2004.

Resultados: Se ha apreciado un aumento en la incidencia de tumores renales en el segundo periodo y de igual manera un aumento en el diagnóstico incidental y en la práctica de cirugía conservadora de parénquima. El tipo histológico más frecuente fue el de células claras en ambos periodos y el tamaño tumoral era mayor en el primer periodo que en el segundo. El estadio tumoral más frecuente fue el I, aunque en el primer periodo se encontró mayor porcentaje de estadios IV. La mortalidad causa-específica ha aumentado en los últimos años.

Conclusión: Se aprecia un aumento en la incidencia de tumores renales y, aunque estos se diagnostican en estadios más precoces, no se ha producido un descenso en la mortalidad.

Palabras clave: Cáncer de células renales. Incidencia. Diagnóstico incidental.

\section{ABSTRACT \\ GROWING INCIDENCE IN RENAL CELL CARCINOMA}

Objectives: To analyze the change in the behavior of renal cell carcinoma for its presentation, treatment, histology and mortality during a 17 year period.

Material and method: Retrospective study on 212 patients diagnosed with renal cell carcinoma in our Department from the year 1988 up to 2004, analyzing the clinical and demographic data and comparing them to each other according to two periods: 1988-1996 and 1997-2004.

Results: An increase has been appreciated in the incidence of renal tumors in the second period and in a same way an increase in the incidental diagnosis and in the practice of nephron sparing surgery. Clear cell type was the most frequent in both periods and tumoral size was higher in the first period than in second. TNM stage I was the most frequent, although in first period it was higher percentage of stage IV. Cause-specific mortality has increased in the last years.

Conclusion: An increase is appreciated in the incidence of renal cell tumors. Although the diagnosis is in earlier stages, a descent in the mortality has not been found. 
$\mathrm{S}_{\mathrm{d}}^{\mathrm{e}}$ estima que en EEUU en el año 2005 se diagnosticarán más de 35.000 nuevos casos de carcinoma de células renales (CCR), de los cuales aproximadamente el $35 \%$ morirán por esta causa $^{1}$. La incidencia del CCR ha aumentado alrededor del 126\% desde 1950, produciéndose un incremento anual progresivo en las tasas de incidencia del $2,3 \%$ al $4,3 \%$, fundamentalmente debido al mayor uso de técnicas de imagen. Sin embargo, paralelamente la mortalidad se ha incrementado en un $36,5 \%$ anual, por lo que la detección incidental por sí sola no explica del todo este aumento de la incidencia ${ }^{2,3}$, por lo que otros factores como los ambientales, dietéticos o genéticos parecen estar también implicados en el creciente diagnóstico de este tipo de tumores que, actualmente, ronda el 3\% de todos los tipos de tumor que afectan al ser humano ${ }^{1,4}$.

Se ha propuesto la ecografía como método de detección precoz de CCR, sobre todo en pacientes con edades superiores a los 50 años, en los que los factores de riesgo son más prevalentes (edad, tabaco, hipertensión, enfermedad familiar predisponente, hemodiálisis crónica, etc.), ya que parece que el diagnóstico precoz produce una sensible mejoría en el pronóstico de estos tumores ${ }^{5}$.

A pesar de todo, parece claro que la historia natural del CCR ha presentado cambios en los últimos años y prueba de ello son los cambios en el comportamiento epidemiológico de este tumor, puesto que junto al aumento de la incidencia y la mortalidad, se ha producido un incremento en la supervivencia, lo cual resulta paradójico ${ }^{2}$.

$\mathrm{El}$ hecho de que no podamos encontrar causas evidentes que expliquen la conducta de este tipo de tumor y que se produzcan variaciones en la presentación del mismo con respecto al pasado nos hace plantearnos el presente trabajo, en el que se pretende analizar algunos aspectos de la epidemiología del CCR en los últimos años y los cambios que se han producido en su manejo clínico.

\section{MATERIAL Y MÉTODO}

Estudio retrospectivo observacional sobre 212 pacientes diagnosticados y tratados quirúrgicamente (realizándose nefrectomía parcial o radical y en algunos casos toma de biopsia por considerarse inoperable) por CCR desde Enero de 1988 hasta Diciembre de 2004 en nuestro servicio.
A partir de las historias clínicas se recogieron datos demográficos y clínicos de dichos pacientes así como de los tumores que presentaban, analizándolos a lo largo de los años y observando los cambios producidos dependiendo del momento en el que fuero diagnosticados. Se valoraron las complicaciones que aparecieron tras la cirugia, de manera que se consideraron 3 tipos de complicaciones: intraoperatorias, las acaecidas durante el acto operatorio; postoperatorias inmediatas, las que ocurrieron dentro de la primera semana tras la cirugía; y, postoperatorias tardías, las que se sucedieron transcurrida una semana de la intervención. Para determinar el grado nuclear se siguió la clasificación de Fuhrman ${ }^{6}$; el tamaño tumoral fue distribuido en 3 categorías: menor de $4 \mathrm{~cm}$, entre 4 y $7 \mathrm{~cm}$, y mayor de $7 \mathrm{~cm}$; y, el estadio tumoral fue ajustado según la última revisión de la clasificación $\mathrm{TNM}^{7}$.

Para el análisis del comportamiento del cáncer renal con el paso de los años se tomó como punto de corte el año 1997, puesto que en ese año se produjo un aumento considerable en el número de casos diagnosticados y representa aproximadamente la mitad del periodo, de manera que fueron comparados los tumores intervenidos desde el año 1988 hasta 1997 y aquéllos operados desde 1997 en adelante.

El análisis estadístico se realizó con el programa informático SPSS 13.0.1, con el que se valoró por medio de tablas de contigencia y tests de comparación de medias el grado de significación entre distintas variables y los periodos considerados.

\section{RESULTADOS}

La edad media de los pacientes fue de 62,01 años (D.E:12,14). De los 212 pacientes, 133 fueron varones $(62,7 \%)$, lo que supone una relación varón/mujer de 1,68. En el periodo 1988-1996 la edad media fue de 60,75 años (D.E: 11,76), siendo varones $34(54,8 \%)$ y la relación varón/mujer 1,2 , mientras que la edad media en el periodo 1997-2004 fue de 62,52 años (D.E: 12,3), con 99 varones (66\%) y una relación varón/mujer de 1,9 (p:n.s.).

El número de pacientes diagnosticados a lo largo de los años ha ido aumentando progresivamente, de manera que de 5 pacientes diagnosticados e intervenidos por tumor renal en 1988 se 
pasó a 28 en el año 2004. Así pues, en el periodo 1988-1996 fueron 62 los pacientes diagnosticados e intervenidos, mientras que desde 1997 a 2004 se trataron 150. De igual manera, el número de cirugías conservadoras de parénquima ha aumentado en números absolutos, puesto que en el primer periodo se realizaron 16 nefrectomías parciales frente a 38 en el segundo. No obstante porcentualmente no se aprecian diferencias significativas, ya que supuso un $25,8 \%$ de 1988 a 1996 y un $25,3 \%$ a partir de 1997 . Estos datos se muestran en la Figura 1.

La cirugía conservadora de parénquima se realizó de manera electiva, es decir, en pacientes con riñón contralateral sano y sin patología acompañante que obligara a la conservación de tejido renal en el 85,5\% de los casos, siendo en el $81,3 \%$ de los casos en el primer periodo y en el $87,2 \%$ en el segundo (p:n.s.).

El tiempo medio que los pacientes permanecieron ingresados por la intervención quirúrgica fue de 9,02 días (D.E: 4,935) con un rango de 1 a 42 días (1 día debido a exitus del paciente). Los pacientes intervenidos entre 1988 y 1996 permanecieron ingresados una media de 10,62 días (D.E: 5,456; Rango: 5-42 días), mientras que los intervenidos a partir de 1997 presentaron una media de ingreso de 8,37 días (D.E: 4,56; Rango: 1-39 días) $(\mathrm{p}=0,02)$.

El motivo por el que se llegó al diagnóstico del tumor fue mayoritariamente por el hallazgo incidental, que se presentó en el 50,5\% de los casos, seguido en frecuencia por la hematuria en un
$22,6 \%$ y por el dolor (14,6\%). En un 10,4\% de los casos se asociaron dos síntomas y tan sólo en 2 pacientes $(0,9 \%)$ la enfermedad se manifestó con la triada clásica de Guyon.

El hallazgo incidental en los pacientes tratados antes de 1997 supuso el 40,3\% de los casos, mientras que en el periodo 1997-2004 fue de un $54,7 \%(\mathrm{p}=0,05)$. Los datos globales y comparativos según el periodo se muestran en la Tabla 1.

Globalmente obtuvimos una tasa de complicaciones intraoperatorias del $14,2 \%$, de postoperatorias inmediatas del $13,7 \%$ y de tardías del $4,7 \%$. Según los periodos considerados, encontramos unas tasas del $22,6 \%, 19,4 \%$ y $8,1 \%$ en el primer periodo y del $10,7 \%, 11,3 \%$ y $3,3 \%$ en el segundo para complicaciones intraoperatorias, postoperatorias inmediatas y postoperatorias tardías respec-

\section{Tabla 1}

Síntoma principal que presentaron los pacientes en el momento del diagnóstico

\begin{tabular}{lcccccc}
\hline & $\mathbf{1 9 8 8 - 2 0 0 4}$ & $\mathbf{1 9 8 8 - 1 9 9 6}$ & $\mathbf{1 9 9 7 - 2 0 0 4}$ \\
\hline $\begin{array}{l}\text { Síntoma } \\
\text { principal }\end{array}$ & $\mathbf{N}$ & $\%$ & $\mathbf{N}$ & $\%$ & $\mathbf{N}$ & $\%$ \\
\hline Masa & 11 & 5,2 & 5 & 8,1 & 6 & 4 \\
Hematuria & 48 & 22,6 & 17 & 27,4 & 31 & 20,7 \\
Dolor & 31 & 14,6 & 13 & 21 & 18 & 12 \\
Incidental & 107 & 50,5 & 25 & 40,3 & 82 & 54,7 \\
Otros & 15 & 7,1 & 2 & 3,2 & 13 & 8,7 \\
\hline Total & 212 & 100 & 62 & 100 & 150 & 100 \\
\hline
\end{tabular}

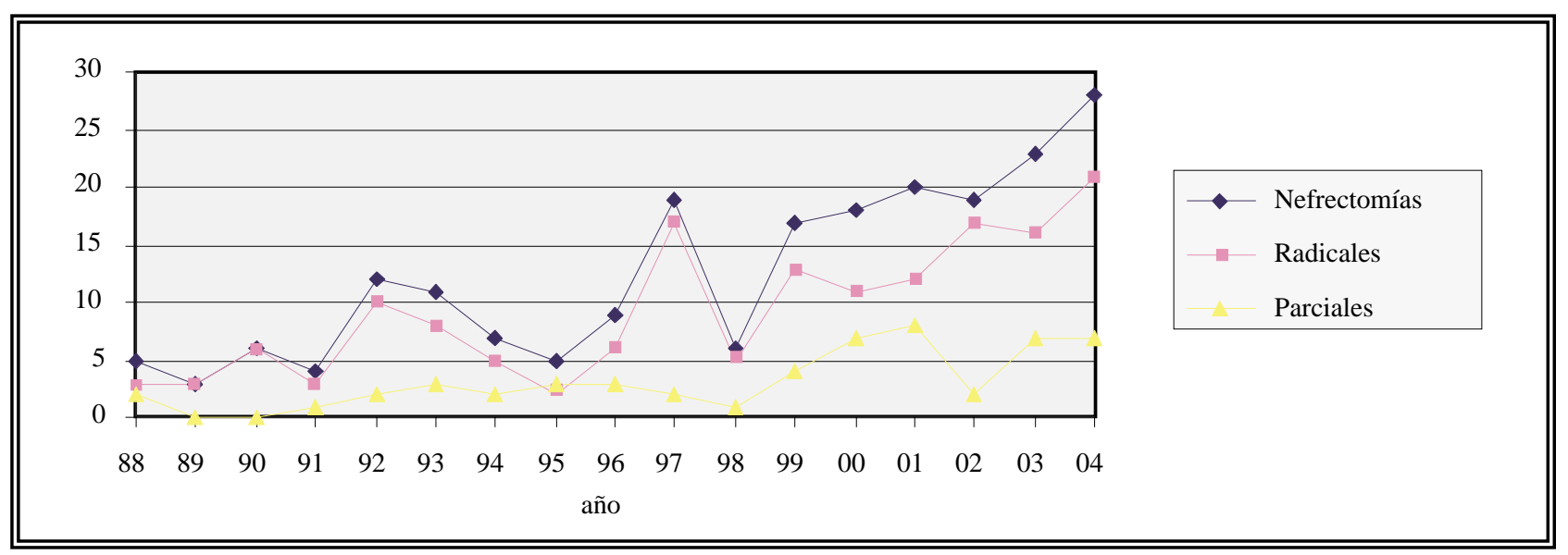

FIGURA 1. Número de nefrectomias realizadas por año indicando el número de cirugias radicales y conservadoras de parénquia 


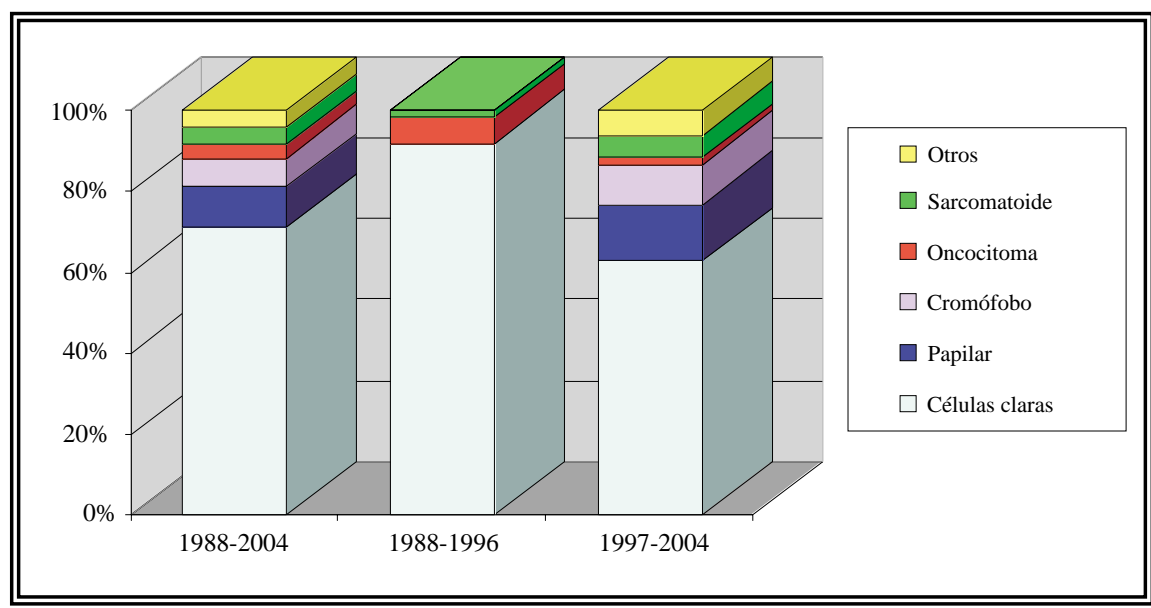

FIGURA 2. Tipo histológico de los tumores.

tivamente. Únicamente se encontraron diferencias significativas entre periodos en las complicaciones intraoperatorias $(\mathrm{p}=0,024)$.

Los tipos histológicos del tumor se muestran en la Figura 2. Se observa que en el periodo 1988-1996 el porcentaje de carcinomas de células claras es alto $(91,9 \%)$ no hallándose ningún tumor papilar, mientras que en el periodo 19972004, los porcentajes están más repartidos, siempre con claro predominio del subtipo de células claras.

En cuanto al grado nuclear, los datos globales se muestran en la Figura 3. El grado 2 es el predominante ya que supone el $54,7 \%$, seguido en orden de frecuencia por los grados 3, 4 y 1. En un $2,8 \%$ no fue posible la catalogación. Estos datos por periodos son similares, ya que el grado 2

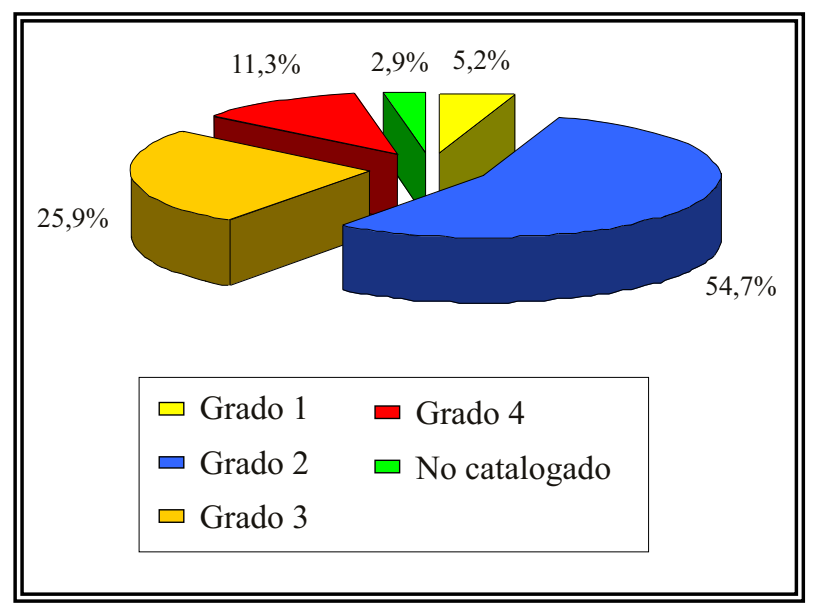

FIGURA 3. Grado nuclear de Fuhrman de los tumores diagnosticados en todo el periodo del estudio. sigue siendo el más frecuente constituyendo el $54,8 \%$ y el $54,7 \%$ para el primer y segundo periodo respectivamente.

De manera global, el tamaño más frecuentemente hallado fue entre 4 y $7 \mathrm{~cm}$. Sin embargo llama la atención como en el primer periodo el grupo de tumores más frecuente fue el de los mayores de $7 \mathrm{~cm}$ $(48,4 \%)$, mientras que los tumores diagnosticados a partir de 1997 presentaron un tamaño mayoritario comprendido entre 4 y $7 \mathrm{~cm}$, apreciándose una tendencia lineal significativa $(p=0,029)$. En cuanto al estadio tumoral observamos que, si bien el estadio I es el más frecuente tanto en el conjunto de todos los tumores como en la división por periodos, llama la atención que el porcentaje de estadios IV es sensiblemente superior en los tumores dentro del primer periodo considerado $(24,2 \%)$ que en el segundo (14,7\%). Todos estos datos se reflejan en la Tabla 2 .

Tabla 2

Tamaño y estadio del tumor distribuidos por periodos

\begin{tabular}{|c|c|c|c|c|c|c|c|}
\hline & & \multicolumn{2}{|c|}{ 1988-2004 } & \multicolumn{2}{|c|}{ 1988-1996 } & \multicolumn{2}{|c|}{ 1997-2004 } \\
\hline & & & $\mathbf{N}$ & $\% \mathbf{N}$ & $\%$ & $\mathbf{N}$ & $\%$ \\
\hline \multirow[t]{4}{*}{ Tamaño } & $<4 \mathrm{~cm}$ & 41 & 19,3 & 8 & 12,9 & 33 & 22 \\
\hline & $4-7 \mathrm{~cm}$ & 91 & 42,9 & 24 & 38,7 & 67 & 44,7 \\
\hline & $>7 \mathrm{~cm}$ & 80 & 37,7 & 30 & 48,4 & 50 & 33,3 \\
\hline & Total & 212 & 100 & 62 & 100 & 150 & 100 \\
\hline \multirow[t]{5}{*}{ Estadio } & I & 100 & 47,2 & 24 & 38,7 & 76 & 50,7 \\
\hline & II & 41 & 19,3 & 16 & 25,8 & 25 & 16,7 \\
\hline & III & 34 & 16 & 7 & 11,3 & 27 & 18 \\
\hline & IV & 37 & 17,5 & 15 & 24,2 & 22 & 14,7 \\
\hline & Total & 212 & 100 & 62 & 100 & 150 & 100 \\
\hline
\end{tabular}

En cuanto al porcentaje de exitus, hasta el momento del estudio, encontramos un total de 53 fallecimientos (25\%), de los cuales $33(62,3 \%)$ fueron debidos a la propia enfermedad tumoral. En el periodo 1988-1996, se produjeron 25 fallecimientos $(40,3 \%)$ siendo 14 de ellos (56\%) debidos al tumor, mientras que en el periodo 1997- 
2004 fueron 28 las muertes $(18,7 \%)$, de las cuales $19(67,9 \%)$ se debieron a la enfermedad tumoral (p:n.s.).

\section{DISCUSIÓN}

Aunque puede aparecer a cualquier edad, el CCR aparece entre los 50 y 70 años en alrededor del $80 \%$ de los casos, con un pico de incidencia en la sexta década de la vida ${ }^{8}$. Es un tumor más frecuente en hombres que en mujeres, con una relación varón/mujer que oscila entre 1,6 y 2 a $1^{9}$, lo que concuerda con los datos obtenidos, sobre todo en el segundo periodo.

El CCR ha mostrado un progresivo incremento de su incidencia en las últimas décadas. Este aumento se ha producido en todos los tipos de tumor, pero fundamentalmente en los tumores localizados $^{2}$, lo que explica, por otra parte, el aumento de la práctica de cirugía conservadora de parénquima, por el diagnóstico de masas más pequeñas y en estadios más precoces ${ }^{10}$. En el presente estudio encontramos un incremento progresivo en la detección de cáncer renal a lo largo de los años, quizá por esta misma razón se produce un aumento en la práctica de cirugía conservadora de parénquima renal, si bien, en nuestra serie este aumento es discreto, probablemente por los criterios de realización, sobre todo en los casos en los que se hace de manera electiva. No obstante, el futuro en el manejo de este tipo de lesiones incidentales y de tamaño reducido se dirige hacia la práctica de procedimientos cada vez menos invasivos ${ }^{11}$.

El hecho de que el mayor incremento de la incidencia de los tumores renales sea a expensas de hallazgos incidentales ha hecho plantear la posibilidad de crear un método de diagnóstico precoz ${ }^{12}$, sin embargo no parece que resulte rentable, puesto que pese a que la ecografía representa un método económico, sensible y específico, hoy en día sólo se recomienda para determinados grupos de riesgo (hemodiálisis prolongada, enfermedad quística renal adquirida, enfermedad de von Hippel-Lindau). Un programa de cribado del cáncer renal mediante ecografía resultaría caro ya que se ha estimado que para cada cáncer descubierto con este método se deberían realizar más de 2.000 estudios ecográficos ${ }^{12,13}$.
Las complicaciones derivadas del tratamiento quirúrgico se deben a la elección de la técnica quirúrgica empleada, fundamentalmente entre cirugía radical y conservadora de parénquima, sin embargo no parece haber una diferencia significativa entre ambos procedimientos, si no contamos el empleo de más tiempo en la cirugía ${ }^{14}$. En la presente serie dos tipos distintos de técnica fueron empleadas, ya que ningún paciente fue tratado mediante cirugía laparoscópica. Encontramos una mayor tasa de complicaciones en el primer periodo considerado, atribuible probablemente a tumores de mayor tamaño y, por tanto, de más difícil abordaje quirúrgico.

El CCR de células claras constituye el subtipo histológico más frecuente dentro de las variantes celulares del carcinoma renal ${ }^{15}$. En el presente estudio, constituye la variante más frecuente tanto de manera global y en los periodos considerados. Llama la atención el no hallarse ningún tumor variante papilar en el primer periodo, ya que la gran mayoría fue CCR de células claras y las restantes variantes halladas fueron el oncocitoma y el tumor con diferenciación sarcomatoide. Este hecho quizá se deba al uso de distintas clasificaciones patológicas y a la aparición de patólogos especialistas en Uropatología en los últimos años en nuestro centro. En el periodo 1997-2004 se encontró un 10\% de tumores cromófobos, lo que es ligeramente superior a la frecuencia descrita clásicamente de estos tumores $^{16}$, aunque se ha descrito un ligero aumento en el diagnóstico de esta variante celular ${ }^{17}$.

$\mathrm{El}$ grado nuclear representa un factor pronóstico importante dentro de los tumores renales. Si bien está sujeto a ciertas controversias en cuanto a su uso, se ha demostrado que, en general, el más frecuentemente hallado es el grado $2^{18,19}$, tal y como ocurre en el presente estudio.

El tamaño tumoral también constituye un factor pronóstico a tener en cuenta en los pacientes diagnosticados de CCR, de hecho, en la clasificación TNM usada actualmente, se realizó una subdivisión en la categoría $\mathrm{T} 1$ en base únicamente al tamaño. Dicha clasificación se debe a la constatación de que la supervivencia sin enfermedad es significativamente mejor en los pacientes con tumores menores de $4 \mathrm{~cm}^{20}$. Además el hecho de que se haya producido un aumento en el diagnóstico precoz de los tumores y por tanto de 
menor tamaño apoya la circunstancia que en el primer periodo de este estudio los tumores presentaran un mayor tamaño, probablemente por un diagnóstico más diferido. Por todo ello, se considera más acertada la subestadificación del estadio $\mathrm{T} 1^{21,22}$.

En cuanto al estadio tumoral, cabe decir que, si bien en ambos periodos los estadios más bajos (I y II) fueron los más frecuentes, sí se encontraron globalmente estadios más altos en el periodo 1988-1996. Este hecho va a favor de que, aunque se ha producido un incremento en el número de tumores diagnosticados en los últimos años, éstos se detectan en estadios más precoces, con menor grado de invasión metastásica, ya sea ganglionar o a distancia y con menor tamaño.

Por esta misma razón, el número de fallecimientos fue porcentualmente mayor en el primer periodo. Sin embargo el porcentaje de muerte causa-específica es mayor en el segundo periodo, lo que constituye una paradoja, pues a pesar de adelantar el momento del diagnóstico no se consigue mejorar la supervivencia, habiendo series en las que incluso empeora ${ }^{2,3,23}$.

En conclusión, el carcinoma renal sigue suponiendo un reto en la práctica diaria del urólogo, puesto que presenta una incidencia creciente. A pesar de contar con mejores armas diagnósticas y terapéuticas hoy en día no ha sido posible rebajar la mortalidad que supone este tipo de tumores, siendo necesario ampliar conocimientos en el impredecible comportamiento del cáncer renal y enfocar su manejo desde distintos puntos de vista con el objeto de conseguir mejores resultados.

\section{REFERENCIAS}

1. Jemal A, Murria T, Ward E, Samuels A, Tiwari RC, Ghafoor A, et al. Cancer statistics, 2005. CA Cancer J Clin 2005;55:10-30.

2. Pantuck AJ, Zisman A, Belldegrun AS. The changing natural history of renal cell carcinoma. J Urol 2001;166:1611-1623.

3. Chow WH, Devesa SS, Warren JL, Fraumeni JF Jr. Rising incidence of renal cell cancer in the United States. JAMA 1999;281:16281631.

4. Uzzo RG, Cairos P, Al-Saleem T, Hudes G, Haas N, Greenberg RE, et al. The basic biology and inmunobiology of renal cell carcinoma: Considerations for the clinician. Urol Clin North Am 2003;30:423436.

5. Sweeney JP, Thornhill JA, Grainger R, McDermott TED, Butler MR. Incidentally detected renal cell carcinoma: pathological features, survival trends and implications for treatment. Br J Urol 1996; 78:351-353.

6. Fuhrman SA, Lasky LC, Limas C. Prognostic significance of morphologic paramenters in renal cell carcinoma. Am J Surg Pathol 1982;6:655-663.
7. Greene FL, Page DL, Fleming ID, Fritz A, Balch CM, Haller DG, et al. AJCC Cancer Staging Manual, $6^{\text {th }}$ ed. New York: Springer Press, 2002;323-328.

8. McLaughlin JK, Lipworth L. Epidemiologic aspects of renal cell cancer. Semin Oncol. 2000;27:115-123.

9. Golbano Ablanque J, Chicharro Alamarza GJ, Otero Tejero I, Serrano Pascual A. Epidemiología, etiología, clínica e historia natural del carcinoma de células renales. En: Oncologia urológica. 1ํㅡㄹ Edición. Resel Estévez L, Moreno Sierra J. Madrid, España. Edit. Grupo Saned, 2003;311-322.

10. Giménez Bachs JM, Salinas Sánchez AS, Hernández Millán IR, Ruiz Mondéjar R, Lorenzo Romero JG, Segura Martín M, et al. Cirugía conservadora de parénquima en los tumores renales. Rev Clin Esp. 2004;204:191-197.

11. Pantuck AJ, Zisman A, Rauch MK, Belldegrun A. Incidental renal tumors. Urology. 2000;56:190-196.

12. Jayson M, Sanders H. Increased incidence of serendipitously discovered renal cell carcinoma. Urology. 1998;51:203-205.

13. Malaeb BS, Martin DJ, Littooy FN, Lotan Y, Waters WB, Flanigan $\mathrm{RC}$, et al. The utility of screening renal ultrasonography: identifying renal cell carcinoma in an elderly asymptomatic population. BJU Int. 2005;95:977-981.

14. Corman JM, Penson DF, Hur K, Khuri SF, Daley J, Henderson W, et al. Comparison of complications alter radical and partial nephrectomy: results from the Nacional Veterans Administration Surgical Quality Improvement Program. BJU Int. 2000;86:782789.

15. Novick AC, Campbell SC. Renal Tumors. En: Campbell's Urology. $8^{\text {th }}$ Edition. Walsh PC, Retik AB, Vaughan DE, Wein AJ. Philadelphia (Pennsylvania), USA. Edit. Saunders Company, 2003:2672-2731.

16. Störkel S, Eble JN, Adlakha K, Amin M, Blute ML, Bostwick DG, et al. Classification of renal cell carcinoma. Workgroup № 1 . Union Internationale contre le cancer (UICC) and the American Joint Committee on Cancer (AJCC). Cancer 1997;80:987-989.

17. Reuter VE, Presti JC: Contemporary approach to the classification of renal epithelial tumors. Semin Oncol. 2000;27:124-137.

18. Medeiros LJ, Jones EC, Aizawa S, Aldape HC, Cheville JC, Goldstein NS, et al. Grading of renal cell carcinoma. Workgroup № 2. Union Internationale contre le cancer (UICC) and the American Joint Committee on Cancer (AJCC). Cancer 1997;80:990-991.

19. Goldstein NS. Grading of renal cell carcinoma. Urol Clin North Am 1999;26:637-642.

20. Leibovich BC, Pantuck AJ, Bui MHT, Ryu-Han K, Zisman A, Figlin $\mathrm{R}$, et al. Current staging of renal cell carcinoma. Urol Clin N Am. 2003;30:481-497.

21. Frank I, Blute ML, Leibovich BC, Cheville JC, Lohse CM, Zincke H. Independent validation of the 2002 American Joint Committee on cancer primary tumor classification for renal cell carcinoma using a large, single institution cohort. J Urol 2005;173:1889-1892.

22. López Luque AJ, Anglada Curado FJ, Carazo Carazo JL, Álvarez Kindelan J, Márqeuz López J, Leva Vallejo M, et al. Revisión del punto de corte entre los estadios T1 Y T2 en la clasificación TNM del carcinoma renal de 1997. Arch Esp Urol. 2003;27:292-296.

23. Ortiz Gorraiz M, Vicente Prados FJ, Rosales Leal JL, Honrubia Vílchez B, Martínez Morcillo A, Cózar Olmo JM, et al. Valoración de factores pronósticos de la supervivencia en una serie de 202 pacientes intervenidos por carcinoma de células renales. Arch Esp Urol. 2005;29:179-189.

Dr. J.M. Giménez Bachs

E-mail: gbjosem@sescam.jccm.es

(Trabajo recibido el 23 de noviembre 2005) 